\title{
Cortical activation evoked by visual mental imagery as measured by fMRI
}

\author{
Markus Knauff, I,CA Jan Kassubek, ${ }^{2}$ Thomas Mulack',2 and Mark W. Greenlee ${ }^{3}$ \\ 'Center for Cognitive Science and ${ }^{2}$ Department of Neurology, University of Freiburg, Friedrichstr. 50, 79098 Freiburg; \\ ${ }^{3}$ Department of Psychology, University of Oldenburg, Germany \\ ${ }^{\mathrm{CA}}$ Corresponding Author
}

Received 12 September 2000; accepted 6 October 2000

\begin{abstract}
One of the major controversies in cognitive neuroscience is whether the primary visual cortex and nearby areas are involved in visual mental imagery. In an fMRI study we examined the brain activity of 10 healthy subjects under different task conditions: in the perception condition subjects saw complex geometrical shapes and had to decide whether other highlighted stimuli fell inside or outside the figure. In the imagery condition subjects saw only the highlighted stimuli and were instructed to imagine the previously studied geometrical shapes to solve the same task. Although the behavioral data show a distance effect that would be expected based on
\end{abstract}

topographically organized mental images, the functional imaging data do not show increased activity in the primary visual cortex in the imagery condition. In the occipital cortex a slightly increased activity was found only in the visual association cortex (BA 19), whereas the highest activation was observed in the parietal cortex (BA 7 and 40). The results of the study do not support the assumption that the primary visual cortex is involved in visual mental imagery, but rather that a network of spatial subsystems and higher visual areas appears to be involved. NeuroReport II:3957-3962 (C) 2000 Lippincott Williams \& Wilkins.

Key words: fMRI; Occipito-parietal pathway; Primary visual cortex; Spatial working memory; Symbolic distance effect; Visual mental imagery

\section{INTRODUCTION}

Visual mental imagery can be defined as the manipulation of visual information that comes not from perception but from memory. Consequently, one of the central research issues on imagery is whether the primary visual cortex and nearby cortical areas are activated by visual mental imagery. The strictest form of imagery theories was introduced by Kosslyn [1], who assumed that during mental imagery the geometrical information of remembered objects and scenes are processed in the primary visual cortex. Indeed, this assumption is supported by a series of PET studies by Kosslyn and colleagues, who found increased blood flow in Brodmann area (BA) 17, corresponding to the primary visual cortex V1, during mental imagery of letters [2] and objects in different sizes [3]. More recent support comes from a combined PET and rTMS study in which subjects compared properties of stripes [4]. Using fMRI, two studies support the hypothesis that topographically organized areas of the visual cortex are evoked by imagery. One study compared sensorimotor and cognitive tasks and reports a significant increase of activation in the primary visual cortex during imagery tasks [5]. The other study (using a $4 \mathrm{~T}$ scanner) reports that the lateral geniculate nucleus was activated during visual imagery processes in the human brain together with V1 and other activation [6].

Other studies, however, did not find evidence for activation in the primary visual cortex during visual imagery. A PET study [7] did not find increased activity in the visual cortex when subjects recalled studied visual patterns. Another series of fMRI studies investigated the cortical activity during mental exploration of two-dimensional [8] and three-dimensional scenes [9], and did not observe significant activation in the primary visual cortex. The same result is reported in fMRI studies, which examined cortical activity during image generation for cued words and found no increased activity in the primary visual cortex [10].

Based on these results, some authors argue that there is a high degree of interaction between mental imagery and other cognitive functions and that no unique cortical area for visual mental imagery can be identified [11].

In the following we report an fMRI study in which subjects perceived geometrical shapes and afterwards had to envisage the same figure to solve a matching task. First we report some behavioral data followed by the functional imaging data. At a first glance, these sets of data seem to be in contradiction to each other. In fact, however, they are only incompatible within the strictest version of visual mental imagery theories, but agree with more moderate approaches.

\section{SUBJECTS AND METHODS}

Subjects: The subjects were 10 healthy male right-handed volunteers (age range 27-41, mean 33 years). None had any history of neurological or ophthalmological disorders. All subjects had normal or corrected-to-normal visual 
acuity. Informed consent was obtained in writing. All subjects participated in a training experiment in which they solved 24 tasks outside the scanner on a computer.

fMRI activation paradigm: The experiment consisted of two repetitions of the experimental run. Each experimental run lasted 369s, during which a 16-slice volume was imaged repeatedly (90 volume acquisitions, $4.1 \mathrm{~s}$ each). The stimulation paradigm consisted of four different blocks: (1) fixation of a cross on neutral background ('off' condition); (2) visualization of a grid (baseline condition BC); (3) visualization of a grid with a dark figure drawn into it (perception condition PC); (4) visualization of an empty grid, now with maintaining the figure from the perception condition in mind and mental imagery of its form and position (imagery condition IC).

A model of the sequence of blocks within one run is shown as an inset in Fig. 1. Visual stimuli were projected onto a rear-projection screen covering the rear end of the scanner bore by an LCD projector. The subject lay on his back in the scanner, looking up into a mirror in which an image of the projection screen was reflected. The basic grid stimulus was circular, consisting of five circles with 10 spokes, thus resulting in 50 grid elements. The figures in PC were presented in dark grey, consisted of 25 connected elements each and were chosen to be as abstract as possible. These figures had to be imagined in IC. Different figures were used in each block.

Subjects' performance was controlled for by an intrascanner behavioral test: one of the grid elements was briefly highlighted every 4.1s (six times during one epoch), and subjects had to click a button as soon as they saw it (BC) or had to decide via button-click if this grid element fell on the perceived figure or not (PC). In IC subjects had to decide if the grid element fell on the imagined figure. In the stimulus material we varied the distance between the highlighted grid element and the figure, resulting in three different groups: inside the figure, outside close (touching the figure at one edge at least) and outside far (at least three grid elements between element and figure). This was done to look for possible distance effects. Examples for the stimulus presentations in one block, together with examples of highlighted grid elements, are given in Fig. 1.

MRI acquisition: Local variations in blood oxygenation level dependent (BOLD) response were measured on a

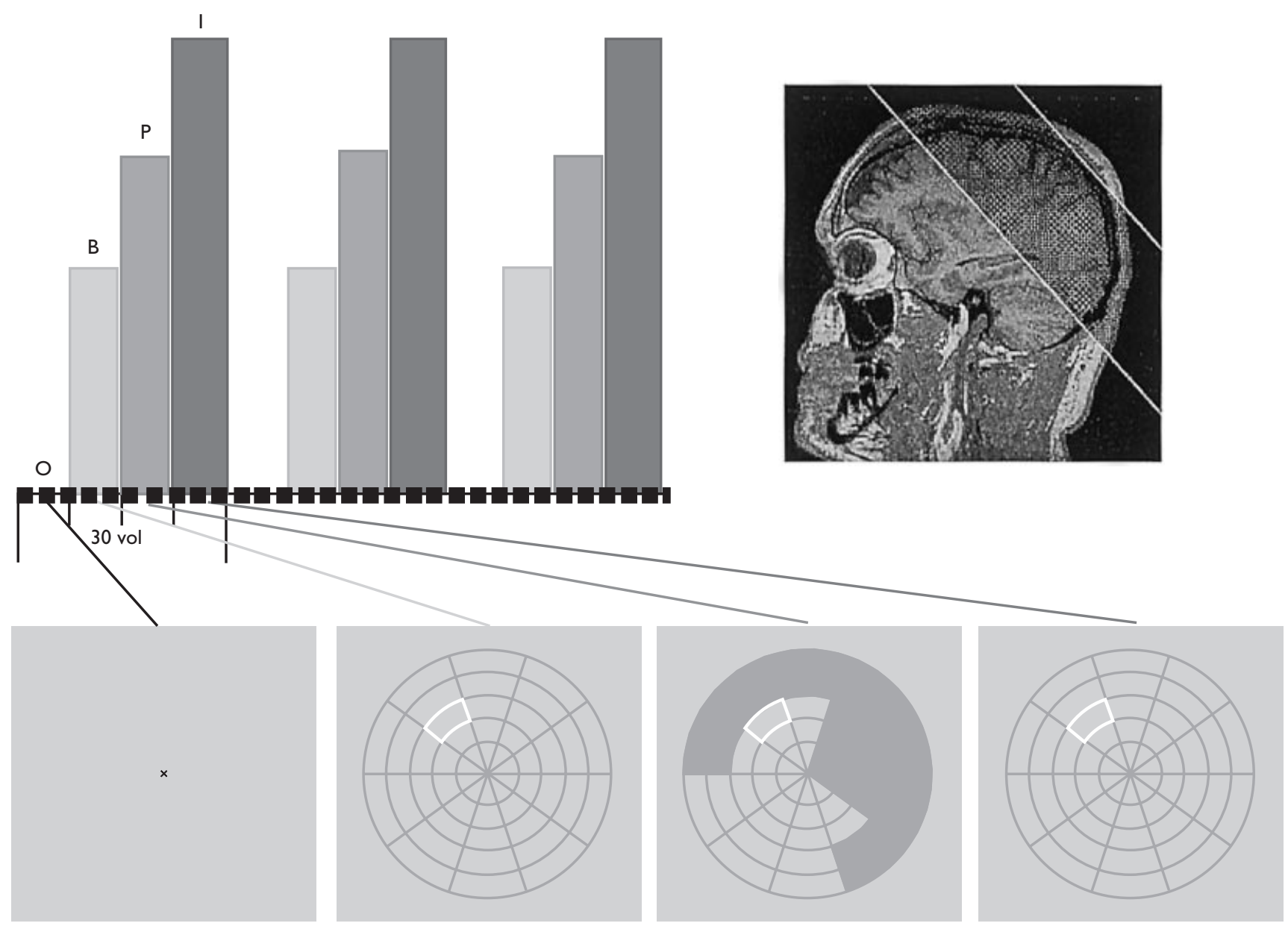

Fig. I. Upper left: block design of an experimental run; upper right: acquisition volume; lower row: examples of the visual stimuli for the experimental conditions off, baseline (BC), perception (PC) and imagery condition (IC), showing a highlighted grid element. 
1.5 T Vision scanner (Siemens) using susceptibility-based functional magnetic resonance imaging, applying gradientrecalled echo-planar imaging (EPI) sequences. Sixteen parallel $4 \mathrm{~mm}$ planes, positioned slightly oblique to the axial plane and covering the parietal and occipital cortex (position of the acquisition volume see Fig. 1, right upper row), were imaged using a T2*-weighted sequence (TR $4100 \mathrm{~ms}$, TE $66 \mathrm{~ms}$, FA $90^{\circ}$, matrix $256 \times 256 \mathrm{~mm}^{2}$, voxel $2 \times$ $\left.2 \times 4 \mathrm{~mm}^{3}\right)$.

Sagittal T1-weighted magnetization-prepared rapidacquisition gradient echo (MP-RAGE) images of the entire brain (160 slices) were acquired for anatomical localization of functional responses (TR $40 \mathrm{~ms}$, TE $6 \mathrm{~ms}$, FA $40^{\circ}$, matrix $256 \times 256 \mathrm{~mm}^{2}$, voxel size $\left.1 \times 1 \times 1 \mathrm{~mm}^{3}\right)$. Statistical maps were transformed to the same resolution as the 3-D MR data set by interpolation. Each individual brain was scaled linearly to match the Talairach atlas [12], and Talairach coordinates are reported for the center of each region of interest (ROI).

Data analysis and statistics: The data were analyzed and visualized using the BrainTools software package. Preprocessing was performed as described in previous publications [13,14]. Functional activation images were constructed as pseudo-color overlays on the corresponding T1-weighted anatomical slices. Only voxels were visualized with correlation coefficients $>0.5 \quad\left(\mathrm{P}_{\text {voxel }}<0.001\right.$ where $\mathrm{P}_{\text {voxel }}=$ probability of a false positive, per voxel). The correlation values were then normalized to a Z-score statistics. Responses in selected ROIs were statistically analyzed to determine the relative magnitude of activation across different stimulus conditions. Voxel clusters containing a minimum of $4 \times 4$ contiguous voxels were selected and for the contrasts only ROIs were investigated in which $>50 \%$ of subjects have shown reliable differences of activation (threshold: $Z \geqslant 2.0$ ). Analysis of variance with repeated measurements was performed on the results of BOLD responses in all ROIs. The activation level was indexed by the standard deviation of the $\mathrm{T}^{*}{ }^{*}$ signal. To weight this activation by the extent to which it is correlated with the stimulus time course, we multiplied the response by the standardized correlation coefficient [14]. Additional statistical analysis (ANOVA for repeated measurements) was performed on the ROI data using SPSS. Z-scores of the functional activations were calculated from correlation values, activation amplitudes and estimated degrees of freedom, separated for hemispheres and Brodmann areas. Behavioral data were analyzed for correctness of decision and reaction times. For all statistical analyses the criterion for significance was set at the 0.05 level, and therefore all reported results are at or beyond this level.

\section{RESULTS}

Behavioral data: Overall, subjects' performance inside the scanner $(86 \%$ correct, $875 \mathrm{~ms})$ was slightly worse than during the training phase outside the scanner $(90 \%$ correct, $850 \mathrm{~ms})$. By analyzing the PC and IC separately, we found $96 \%$ correct reactions in PC and $85 \%$ in IC inside the scanner and $90 \%$ and $80 \%$ during the training, respectively. In the following, only the data from inside the scanner are reported, because the pattern of results in the training was almost identical.
The percentages of correct reactions were modulated by the distance between the figure and the highlighted grid element: if the element was nearby the figure, subjects made more errors than when it was far away. However, this difference is statistically significant only for IC (Wilcoxon test; $Z=2.527 ; p<0.012$ ), whereas it did not reach statistical significance for PC (Wilcoxon test; $Z=1.141$; $p>0.15$; Table 1).

The analysis of response latencies for PC and IC shows a similar pattern of results. Again, subjects' performance was modulated by the distance between the figure and the highlighted element: when the element was nearby, the response took more time than when it was far away (IC: $1124 \mathrm{~ms}$ vs $807 \mathrm{~ms}$; PC: $775 \mathrm{~ms}$ vs $767 \mathrm{~ms})$. These differences are statistically reliable for for IC $(t$-test; $t=7.89 ; p<0.001)$, whereas it is not reliable for PC ( $t$-test, $t=-0.243$; $p>0.809$; Table 1).

Because these results fit exactly with what one would expect based on visual mental imagery, we can assume that subjects indeed solved the tasks based on such a strategy.

Functional imaging data: Statistical significance was reached for the main effect of the experimental conditions off, BC, PC and IC $(\mathrm{F}(3)=25.131, p<0.001)$ and the interaction between experimental condition and ROIs $(F(3,15)=8.829, p<0.001)$, whereas there was no significant interaction between hemispheres and experimental conditions $(\mathrm{F}(3)=1.496, p>0.21)$.

Subsequently, we analysed the ROIs separately. In the $\mathrm{PC}$ vs BC contrast an increase in activation was found in the primary visual cortex (BA 17) and in the visual association cortex (BA 18 and BA 19). In all these ROIs the $Z$-scores were above the threshold of $Z \geqslant 2.0$.

The most important result is that in the contrast IC vs BC only two out of 10 subjects showed activation in V1 (GOI and cuneus, BA 17) and that the Z-scores were very low. Thus, there was no significant increase in activation in the primary visual cortex under the imagery condition compared to the baseline condition. The activated ROIs, number of subjects $(n)$ showing activation and mean $Z$ scores for the contrast IC vs BC are presented in Table 2.

Significantly increased activation was found in the superior parietal lobule (SPL) and parts of the precuneus $(\mathrm{PCu})$, summarized as BA 7, and in the inferior parietal lobe (IPL, BA 40). Furthermore, BOLD effects were found in the medial and inferior occipital lobe (GOM and GOI), the human homologs of V2 and V3 corresponding approximately to BA 18 and 19, respectively. However, statistical analysis of amplitudes of activation revealed high correlation only for the parietal areas BA 7 and BA 40 and the

Table I. Percentages of correct response and response latencies in PC and IC as a function of the distance between highlighted element and figure. Only the differences in IC are statistically significant.

\begin{tabular}{lll}
\hline Correct response & Nearby & Far \\
\hline Perception (PC) & $97 \%$ & $99 \%$ \\
Imagery (IC) & $775 \mathrm{~ms}$ & $767 \mathrm{~ms}$ \\
& $78 \%$ & $98 \%$ \\
& $1124 \mathrm{~ms}$ & $807 \mathrm{~ms}$ \\
\hline
\end{tabular}


Table 2. Activated ROIs (anatomical localizations and Brodmann area together with mean Talairach coordinates, separated for the left and right hemisphere), number of subjects $N$ showing $Z \geqslant 2.0$ activation in the contrast IC vs. BC, and mean $Z$-scores.

\begin{tabular}{|c|c|c|c|c|c|c|c|}
\hline \multirow[t]{2}{*}{ Localization } & \multirow[t]{2}{*}{ BA } & \multirow{2}{*}{$\begin{array}{l}\text { Hemisphere } \\
(\mathrm{R} / \mathrm{L})\end{array}$} & \multirow[t]{2}{*}{$n(/ 10)$} & \multicolumn{3}{|c|}{ Mean Talairach coordinates of centers of activation } & \multirow{2}{*}{$\begin{array}{l}\text { Mean Z-score } \\
\text { (IC vs BC) }\end{array}$} \\
\hline & & & & $x$ & y & z & \\
\hline \multirow[t]{2}{*}{ Superior parietal lobule/precuneus } & 7 & $\mathrm{R}$ & 9 & 14 & -76 & 45 & 2.4 \\
\hline & & L & 8 & -11 & -75 & 46 & 2.5 \\
\hline \multirow[t]{2}{*}{ Gyrus occipitalis inferior/cuneus } & 17 & $\mathrm{R}$ & 2 & 4 & -94 & -7 & 0.0 \\
\hline & & L & 0 & -14 & -92 & -1 & 0.5 \\
\hline \multirow[t]{2}{*}{ Gyrus occipitalis inferior } & 18 & $\bar{R}$ & I & 25 & -92 & -7 & 0.4 \\
\hline & & L & 2 & -26 & -89 & -3 & 0.9 \\
\hline \multirow{2}{*}{$\begin{array}{l}\text { Gyrus occipitalis medius/gyrus } \\
\text { temporalis inferior }\end{array}$} & 19 & $\bar{R}$ & 6 & 26 & -86 & 24 & 2.2 \\
\hline & & L & 6 & -28 & -81 & 27 & 2.4 \\
\hline \multirow[t]{2}{*}{ Inferior parietal lobe } & 40 & $\mathrm{R}$ & 4 & 44 & -46 & 47 & 2.1 \\
\hline & & L & 7 & $-4 \mid$ & -49 & 48 & 2.3 \\
\hline
\end{tabular}

visual association area BA 19 in IC $v s$ BC, but not for BA 18 (nor for BA 17 as mentioned before; cf. Z-scores in Table 2 ). The mean activities in the relevant ROIs for the contrast $\mathrm{BC}$ vs IC are depicted in Fig. 2. Fig. 3 shows representative axial and coronal slices showing localization of activated ROIs superimposed on anatomical MRI data sets.

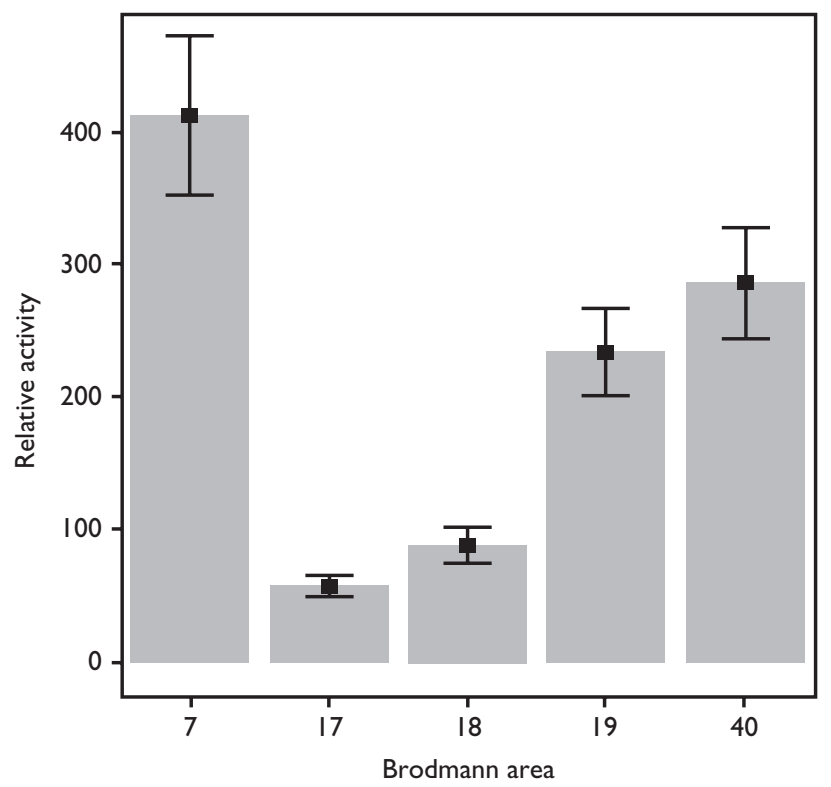

Fig. 2. Relative activation level of the analysed ROls (Brodmann areas $7,17,18,19$ and 40 ) in the contrast BC vs. IC. Values are indexed by the standard deviation of the $\mathrm{T}^{*}$ signal multiplied by the standardized correlation coefficient. The results are pooled for both hemispheres and show the mean values for 10 subjects.

\section{DISCUSSION}

The main issue that led us to conduct the present study was to determine whether the primary visual cortex and nearby areas of the brain are involved in visual mental imagery. If so, there should not only be increasing activity in BA 17 (and nearby areas) under perception conditions but also under imagery conditions.
The first important result derives from the behavioral data. These data show exactly what would be expected based on topographically organized mental images. What we observed is the well-known symbolic distance effect, namely that decisions are more difficult if the objects are nearby, if they have similar sizes etc. [15]. Subjects made more errors and the response took more time if the highlighted objects were located nearby the geometrical figure than when it was far away. Interestingly, this result was much more robust (and statistically significant) under the imagery condition than when subjects directly perceived the figure. However, the main point in the literature is that such distance effects have been taken as evidence that people construct topographically organized mental images and scan them to solve a given task [16]. (This effect should not be confused with the prominent results from Kosslyn's map scanning experiment, which is a completely different task [17].)

However, the interpretation that our subjects constructed topographically organized mental images in primary visual cortex is not supported by the functional imaging data. In fact, we found (slightly) increased activity in BA 17 during imagery conditions for only two of 10 subjects and the Z-scores were very low. This result is in agreement with other studies that also failed to find such activity [7-10].

The results suggest the existence of a conflict: on the one hand the behavioral data is in perfect agreement with the imagery literature in cognitive psychology. On the other hand, the imagery interpretation is not supported by the observed brain activities.

If imagery sensu stricto is not the appropriate interpretation, how else can the performance of our subjects and the observed distance effect be explained? At this point it is necessary to come back to the (bilaterally) increased activity in the superior parietal lobule and precuneus, summarized as BA 7, and in the inferior parietal lobe (IPL, BA 40). Behavioral data $[18,19]$, as well as neuropsychological [20] and brain imaging studies [21], indicate that spatial and object information are computed in dissociable subsystems and this seems to be the case for perception as well as for higher cognitive functions. According to this framework parietal areas are part of the dorsal occipitoparietal pathway [22], and it was shown that they are 




Fig. 3. Representative axial and coronal slices of one subject showing localization of activated ROls in parietal and occipital areas superimposed on anatomical MRI data set. Slice positions according to the Talairach atlas are given at the bottom of slices.

activated during spatial perception [23], spatial attention [24] and in spatial working memory [21].

Indeed, the behavioral data obtained in the present study can be explained as a result of purely spatial processes or representations were. According to this interpretation the presented figures were maintained in spatial working memory, and no involvement of the primary visual cortex was necessary. In this context it was recently argued that such results do not falsify the theory that the primary visual cortex is involved in imagery [25]. The authors argue that most of the studies that failed to find such activity used spatial tasks, whereas none of the studies that found activity in the primary visual cortex used such tasks. Accordingly, the authors believe that the involvement of early visual areas depends on the spatial resolution of the tasks. As mental images become increasingly vivid the authors think they come more and more closely to percepts, resulting in V1 activity [25]. However, from our point of view this cannot provide a satisfying explanation for the obtained pattern of results, because the materials were extremely complex shapes that had to be processed with a high spatial resolution to solve the tasks. One could argue that the source of activity in the spatial areas of the brain is due to the stimulus presentations in a grid. Maybe in this case subjects have a tendency to encode the figures as positions of shaded elements in a grid. However, this explanation is unlikely based on the behavioral data and moreover from the perspective of working memory capacity. If it was true, subjects would encode 25 positions in the grid, a number that far exceeds what is known about spatial working memory capacity [18].

\section{CONCLUSION}

The behavioral data of the present study support the assumption that subjects indeed solved the tasks with a strategy that can be called mental imagery on the cognitive level. Nevertheless, we did not find any significant activation in the primary visual cortex. Instead, we found significantly increased activation in the parietal cortex (BA
7 and 40), as well as in the visual association cortex (BA19). From these results we conclude first that the primary visual cortex is not necessarily involved in visual mental imagery. This result is in agreement with the majority of published studies on visual imagery. Second, a high spatial resolution of the envisaged figures does not support the involvement of early visual areas. The main advantage of the present study is that the used materials consisted of very complex geometrical shapes that, if any, should favor vivid visual images. Third, higher visual areas and the occipito-parietal pathway of the human brain play a central role in visual mental imagery. These regions are not only well known for being responsible for the processing and encoding of spatial information (from perception and from working memory) but also as an interface between visual processes and other cognitive functions.

\section{REFERENCES}

1. Kosslyn SM. Image and Brain. Cambridge, MA: MIT-Press; 1994.

2. Kosslyn SM, Alpert NM Thompson et al. J Cogn Neurosci 5, 263-287 (1993).

3. Kosslyn SM, Thompson WL and Alpert NM. Neuroimage 6, 320-334 (1997).

4. Kosslyn SM, Pascual-Leone A, Felician O et al. Science 284, 167-170 (1999).

5. Sabbah P, Simond G, Levrier O et al. Eur Neurol 35, 131-136 (1995).

6. Chen W, Kato T, Zhu XH et al. Neuroreport 9, 3669-3674 (1998).

7. Roland PE and Gulyas B. Cerebr Cortex 5, 79-93 (1995).

8. Mellet E, Tzourio N, Denis M et al. J Cogn Neurosci 7, 433-445 (1995).

9. Mellet E, Tzourio N, Crivello F et al. J Neurosci 16, 6504-6512 (1996),

10. D'Esposito M, Detre JA, Aguirre GK et al. Neuropsychologia 35, 725-730 (1997).

11. Mellet E, Petit L, Mazoyer B et al. Neuroimage 8, 129-139 (1998).

12. Talairach J and Tournoux P. Co-Planar Atlas of the Human Brain: 3Dimensional Proportional System. Stuttgart: Thieme; 1988.

13. Kassubek J, Schmidtke K, Kimmig H et al. Cogn Brain Res (in press).

14. Smith AT, Greenlee MW, Singh KD et al. J Neurosci 18, 3816-3830 (1998).

15. Banks WP and Flora J. J Exp Psychol: Hum Percept Perf 3, 178-290 (1977).

16. Kosslyn SM. Image and Mind. Cambridge, MA: Harvard University Press; 1980.

17. Kosslyn SM, Ball TM and Reiser BJ. J Exp Psychol: Hum Percept Perf 4, 47-60 (1978). 
18. Logie RH. Visuo-spatial Working Memory. Hove: Lawrence Erlbaum; 1995.

19. Knauff M, Rauh R, Schlieder C et al. Räumliches Denken unter Arbeitsgedächtnisbelastung. (Spatial Thinking under working memory load). In: Schröger E, Mecklinger A and Widmann A, eds. Experimentelle Psychologie. Beiträge zur 41. Tagung experimentell arbeitender Psychologen. Lengerich: Pabst Science Publishers; 1999, pp. 183-184.

20. Pigott $S$ and Milner B. Neuropsychologia 32, 969-981 (1994).

21. Smith EE, Jonides J, Koeppe RA et al. J Cogn Neurosci 7, 337-356 (1995).
22. Ungerleider LG and Mishkin M. Two cortical visual systems. In: Ingle DJ, Goodale MA and Mansfield RJW, eds. Analysis of Visual Behaviour. Cambridge, MA: MIT Press; 1982, pp. 549-586.

23. Posner MI and Peterson SE. Annu Rev Neurosci 13, 25-42 (1990).

24. Haxby JV, Grady CL, Horwitz B et al. Proc Natl Acad Sci USA 88, 16211625 (1991).

25. Mellet E, Tzourio-Mazoyer N, Bricogne S et al. J Cogn Neurosci 2, 98-109 (2000). 